\title{
Coding and Counting Arrangements of Pseudolines
}

\author{
Stefan Felsner • Pavel Valtr
}

Received: 3 November 2009 / Revised: 21 September 2010 / Accepted: 17 January 2011 /

Published online: 9 July 2011

(C) Springer Science+Business Media, LLC 2011

\begin{abstract}
Arrangements of lines and pseudolines are important and appealing objects for research in discrete and computational geometry. We show that there are at most $2^{0.657 n^{2}}$ simple arrangements of $n$ pseudolines in the plane. This improves on previous work by Knuth who proved an upper bound of $3\left(\begin{array}{c}n \\ 2\end{array}\right) \cong 2^{0.792 n^{2}}$ in 1992 and the first author, who obtained $2^{0.697} n^{2}$ in 1997 . The argument uses surprisingly little geometry. The main ingredient is a lemma that was already central to the argument given by Knuth.
\end{abstract}

Keywords Combinatorial geometry $\cdot$ Enumeration $\cdot$ Pseudoline $\cdot$ Cutpath

\section{Introduction}

Arrangements of pseudolines are the topic of a chapter in the Handbook on Discrete and Computational Geometry [8]. The monograph [4] is another general reference. In most texts arrangements of pseudolines are defined with the real projective plane as ambient space. In contrast, we consider arrangements in the Euclidean plane.

A pseudoline in the Euclidean plane is a curve extending to infinity on both sides. An arrangement of pseudolines is a family of pseudolines with the property that each pair of pseudolines has a unique point of intersection where the two pseudolines cross. An arrangement is simple if no three pseudolines have a common point of

\footnotetext{
S. Felsner $(\bowtie)$

Institut für Mathematik, Technische Universität Berlin, Strasse des 17. Juni 136, 10623 Berlin, Germany

e-mail: felsner@math.tu-berlin.de

P. Valtr

Department of Applied Mathematics and Institute for Theoretical Comp. Sci. (ITI), Charles

University, Malostranské nám. 25, 11800 Praha 1, Czech Republic
} 
intersection. Arrangements with a distinguished unbounded cell called the north-cell are known as marked arrangements. Note that if in an arrangement a north-cell $c_{n}$ has been selected, then there is a unique unbounded cell separated from $c_{n}$ by all pseudolines this cell is called the south-cell. Pseudolines in a marked arrangement have a natural orientation such the north-cell is to left and the south-cell to the right of the oriented pseudoline.

Two arrangements are isomorphic, i.e., considered the same, if they can be mapped onto each other by a homeomorphism of the plane. In the case of marked arrangements it is required that an isomorphism respects the distinguished cell and preserves the induced orientations.

In this paper we are interested in the number $B_{n}$ of marked simple arrangements of $n$ pseudolines. It is known, e.g. from [3], that $B_{n} \in 2^{\Theta\left(n^{2}\right)}$. Our interest is in the multiplicative constant $b$ hidden in the $\Theta\left(n^{2}\right)$, i.e., such that $B_{n} \in 2^{b n^{2}+o\left(n^{2}\right)}$. Knuth [9] considers the counting problem for several related classes of arrangements, e.g. arrangements without marking, projective arrangements or more abstractly reorientation classes of uniform oriented matroids of rank three. Their numbers only differ by lower order factors; more precisely, their number is also of the form $2^{b n^{2}+o\left(n^{2}\right)}$ with the same constant $b$.

We are going to study the growth of $b_{n}=\log _{2}\left(B_{n}\right)$. An easy lower bound construction is given in [11, Sect. 6.2]; it yields $b_{n}>\frac{1}{9} n^{2}$. Knuth [9, p. 37] shows $b_{n}>\frac{1}{6} n^{2}-O(n)$. In Sect. 4 we use enumeration results for rhombic tilings to prove $b_{n}>0.188 n^{2}$.

The upper bound $B_{n} \leq 3\left(\begin{array}{l}n \\ 2\end{array}\right)$, i.e., $b_{n} \leq 0.7924 n^{2}$, was shown by Knuth [9, p. 39]. At the end of this monograph, Knuth [9, p. 96] comments that an improved bound of $b_{n} \leq 0.7194 n^{2}$ can be obtained from the sharpest version of the zone theorem. Felsner [3] obtained the bound $b_{n} \leq 0.6974 n^{2}$. In Sect. 2 we review the idea in Knuth's proof and add a new simple idea to get $b_{n} \leq 0.6609 n^{2}$. In Sect. 3 we refine the analysis and prove the bound $b_{n} \leq 0.6571 n^{2}$.

There are several nice representations and encodings of simple arrangements of pseudolines. We close the introduction by explaining three of them. First, however, we fix a labeling of the pseudolines. Given a marked arrangement $\mathcal{A}$ of $n$ pseudolines we label the pseudolines with $1, \ldots, n$ such that an oriented curve from the southcell to the north-cell that has all crossings of pseudolines on the right intersects the pseudolines in increasing order, see Fig. 1(left).

Local sequences Associate with pseudoline $i$ the permutation $\alpha_{i}$ of $\{1, \ldots, n\} \backslash i$ reporting the order from left to right in which the other pseudolines cross line $i$. The family $\left(\alpha_{1}, \alpha_{2}, \ldots, \alpha_{n}\right)$ is called the family of local sequences of the arrangement.

Wiring diagrams Goodman [7] introduced a class of drawings of arrangements called wiring diagrams to get well-arranged pictures of arrangements. The idea is to specify a set of $n$ horizontal lines (wires) and confine the pseudolines to these wires except for positions where they cross each other. In the case of a simple arrangement crossings always involve two pseudolines from adjacent wires. Figure 1 shows an example. 

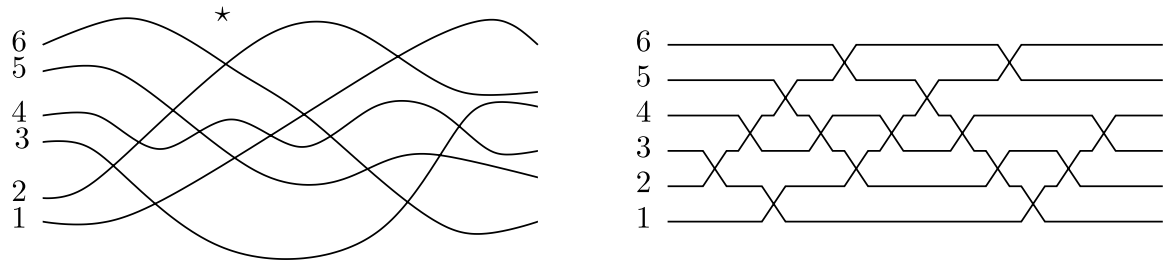

Fig. 1 An arrangement $\mathcal{A}$ with a cell marked by a star and a wiring diagram of $\mathcal{A}$. The local sequences of this arrangement are: $\alpha_{1}=3,5,4,6,2, \alpha_{2}=3,4,5,6,1, \alpha_{3}=2,1,6,5,4, \alpha_{4}=2,5,1,6,3$, $\alpha_{5}=2,4,1,6,3, \alpha_{6}=2,1,4,5,3$
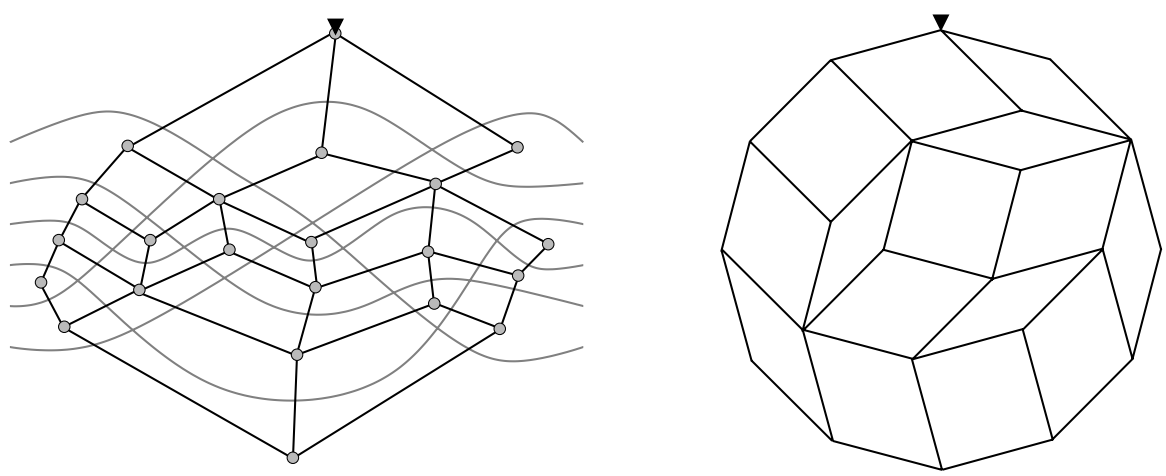

Fig. 2 Arrangement $\mathcal{A}$ with its dual and the corresponding zonotopal tiling

Zonotopal tilings A simple zonotopal tiling $\mathcal{T}$ is a tiling of a regular $2 n$-gon with vertices $x_{0}, x_{1}, \ldots, x_{2 n-1}$ in clockwise order starting with the highest vertex $x_{0}$. The tiles of $\mathcal{T}$ are rhombi $R(i, j), 1 \leq i<j \leq n$, such that $R(i, j)$ has one side which is a translated copy of the segment $\left[x_{i-1}, x_{i}\right]$ and one side which is a translated copy of the segment $\left[x_{j-1}, x_{j}\right]$. The tiles are not allowed to be rotated.

Simple zonotopal tilings can be viewed as normalized drawings of the duals of marked simple arrangements. Figure 2 shows an example. For additional information on zonotopal tilings and their relation to arrangements see [4] and [2].

Proofs of equivalence of the three representations are detailed in [4]. The basic tool for the proof of equivalence is to sweep a representation, resp. the arrangement, from left to right to transform one representation into another.

\section{The Upper Bound}

The upper bound for the number of simple Euclidean arrangements given in [3] was based on 'horizontal encodings' of arrangements. The first step was to replace the numbers in the local sequences $\alpha_{i}$ by single bits, a 1 for numbers $j$ with $j<i$ and a 0 for $j>i$.

The proof of Knuth [9] takes a 'vertical' approach. Let $\mathcal{A}$ be an arrangement of $n+1$ pseudolines and consider pseudoline $n+1$ drawn into the wiring diagram of 

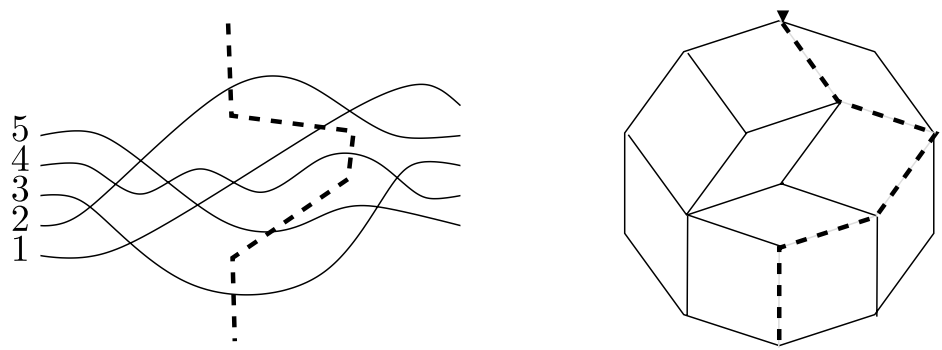

Fig. 3 The cutpath corresponding to pseudoline 6 in the arrangement of pseudolines 1, 2, 3, 4, and 5

Fig. 4 Wiring diagrams of the bubblesort arrangements of six and seven lines
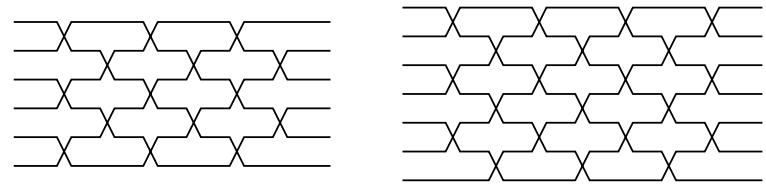

the arrangement $\mathcal{A}^{\prime}$ induced by the first $n$ pseudolines of $\mathcal{A}$. The course of pseudoline $n+1$ describes a cutpath descending from the north-cell to the south-cell of $\mathcal{A}$. Looking at the zonotopal tiling representation of $\mathcal{A}^{\prime}$ as a graph a cutpath corresponds to a vertically decreasing path from the highest vertex $x_{0}$ to the lowest $x_{n}$. Figure 3 shows an example.

The number of arrangements $\mathcal{A}$ such that $\mathcal{A} \backslash\{n+1\}$ equals $\mathcal{A}^{\prime}$ is exactly the number of different cutpaths of $\mathcal{A}^{\prime}$. Define $\gamma_{n}$ as the maximal number of cutpaths an arrangement of $n$ pseudolines can have. It then follows that

$$
B_{n+1} \leq \gamma_{n} \cdot B_{n}
$$

Knuth proves that $\gamma_{n} \leq 3^{n}$ and he also notes that the 'bubblesort arrangement' (see Fig. 4) of size $n$ has approximately $n 2^{n-2}$ cutpaths. Knuth also conjectures that the bubblesort arrangement is the maximizing example. The bubblesort arrangement is a particular Euclidean arrangement corresponding to the projective cyclic arrangement, cf. [12].

In social choice theory a set $T$ of permutations of $[n]$ is called an acyclic set if for all $i, j, k \in[n]$ at most two of $i j k, j k i, k i j$ appear as a restriction of a permutation in $T$ to $\{i, j, k\}$. The interest in acyclic sets comes from the fact that they avoid Condorcet cycles. That is, if voters are constrained to preference lists from an acyclic set $T$, then the majority digraph on the alternatives is acyclic. It has been shown in [6] that the set of cutpaths of an arrangement $\mathcal{A}$ is an acyclic set. Fishburn [5] introduced the alternating scheme as a large acyclic set. It turned out that the permutations in the alternating scheme correspond to the cutpaths of the bubblesort arrangement (Fig. 4). Galambos and Reiner [6] gave a precise formula for the size of the alternating scheme and conjecture that this is the largest size of an acyclic set that can be obtained as the set of cutpaths of an arrangement, i.e., in a different context they came up with the same conjecture as Knuth. Ondřej Bílka, a student of the second author, found a contruction of arrangements with $2.076^{n}$ cutpath [1]. This disproves the conjecture. 


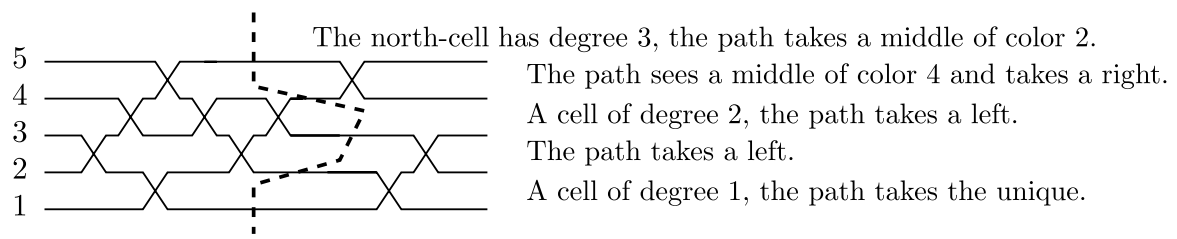

Fig. 5 A cutpath through a wiring diagram

Fig. 6 Illustrating the proof of Lemma 1

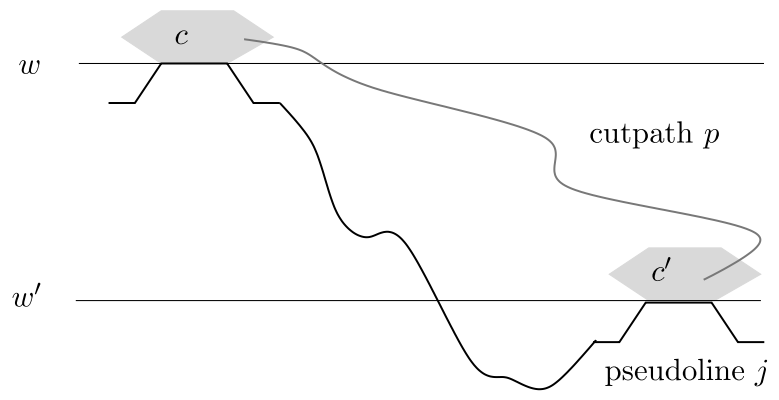

In the remainder of this section we present the main lemma of Knuth and show how to use it to bound the number $\gamma_{n}$ of cutpaths of an arrangement.

Consider a cutpath $p$ descending through the wiring diagram of $\mathcal{A}$. Having reached a cell $c$, the path has to continue by crossing the wire $w$ bounding $c$ from below. The cells that can be reached from $c$ by crossing $w$ are ordered from left to right as $c_{1}, c_{2}, \ldots, c_{d}$. Let their number $d$ be the degree of $c$. When $d \geq 2$ we let $c_{1}$ be the left successor of $c$, and $c_{d}$ be the right successor of $c$. The other cells $c_{2}, \ldots, c_{d-1}$ are called middle successors of $c$. When $d=1$ we let $c_{1}$ be the unique successor of $c$.

When the cutpath $p$ of $\mathcal{A}$ traverses a cell $c$ such that there is a middle successor cell $c^{\prime}$ of $c$ separated from $c$ by pseudoline $j$ we say that $p$ sees a middle of color $j$ at $c$. If $p$ descends from $c$ to $c^{\prime}$ we say that $p$ has crossed pseudoline $j$ as a middle. Figure 5 illustrates the terminology. The middles of $p$ denotes the set of all pseudolines crossed by $p$ as a middle. Similarly, the uniques of $p$ denotes the set of all pseudolines crossed by $p$ when leaving cells of degree 1 .

Lemma 1 (Knuth) For every pseudoline $j$ and every cutpath $p$ it holds: $p$ sees a middle of color $j$ at most once.

Proof Suppose a cutpath $p$ sees a middle of color $j$ at different cells $c$ and $c^{\prime}$. Assuming that $p$ visits $c$ before $c^{\prime}$ we have a situation as sketched in Fig. 6. Let $t$ be the number of wires strictly between $w$ and $w^{\prime}$. Between the visits of the borders of $c$ and $c^{\prime}$, pseudoline $j$ has to change at least $(t+1)+2$ times from a wire to another, i.e., pseudoline $j$ has at least $t+3$ crossings in this range. Every pair of pseudolines has only one crossing. Therefore, every pseudoline crossing $j$ between $c$ and $c^{\prime}$ also has to be traversed by the cutpath $p$ on its way from $c$ to $c^{\prime}$. The cutpath $p$ only intersects $t+1$ wires on its way from $c$ to $c^{\prime}$, a contradiction. 
We use the lemma to encode cutpaths of an arrangement $\mathcal{A}$. With a cutpath $p$ we associate two combinatorial objects:

- A set $M_{p} \subset[n]$ consisting of all $j$ such that pseudoline $j$ is crossed by $p$ as a middle.

- A binary vector $\beta_{p}=\left(b_{p}(0), b_{p}(1), \ldots, b_{p}(n-1)\right)$ such that $b_{p}(i)=1$ only if $p$ proceeds to the left successor of the cell $c$ enclosed between wire $i$ and wire $i+1$.

The cutpath $p$ from Fig. 5 has $M_{p}=\{2\}, \beta_{p}(1)=0$ and $\beta_{p}(2)=\beta_{p}(3)=1$, the values of $\beta_{p}(0)=\beta_{p}(4)=0$ are irrelevant since $p$ is taking a middle in the first step and a unique in the last.

Claim 1 The mapping $p \rightarrow\left(M_{p}, \beta_{p}\right)$ is injective from cutpaths of $\mathcal{A}$ to pairs consisting of a subset $M$ of $[n]$ and a binary vector of length $n$.

Proof Given $\left(M_{p}, \beta_{p}\right)$ the cutpath can uniquely be reconstructed: Assume an initial piece of $p$ up to some cell $c$ has been constructed. If there is only one successor $c^{\prime}$ of $c$, i.e., if the degree of $c$ is one, then $p$ has to continue to $c^{\prime}$. If there is a $j \in M_{p}$ such that $c$ has a middle of color $j$, then $p$ has to cross pseudoline $j$ when leaving $c$ (here we use Lemma 1). Otherwise $c$ has to continue to the left or the right successor of $c$ and a lookup at the corresponding position of $\beta_{p}$ reveals which is to be taken.

From the claim it immediately follows that $\gamma_{n} \leq 2^{n} 2^{n}=4^{n}$. To improve the bound we use two simple observations:

- Every $j$ taken as a middle forces that some entry of $\beta_{p}$ is irrelevant, i.e., not needed for the encoding of the cutpath.

- The lookups of entries of $\beta_{p}$ are done in increasing order of indices.

It follows that we can take $\beta_{p}$ to be a binary string of length $n-\left|M_{p}\right|$ and agree that lookups are always taken at the first unused position of $\beta$. This improved encoding yields:

$$
\gamma_{n} \leq \sum_{k=0}^{n}\left(\begin{array}{l}
n \\
k
\end{array}\right) 2^{n-k}=2^{n}\left(1+\frac{1}{2}\right)^{n}=3^{n} .
$$

This is the upper bound of Knuth, only the arithmetic in our derivation is simpler.

Note that our estimate for the length of $\beta_{p}$ does not yet take into account that some cells may have degree one. Define $\Gamma_{\mathcal{A}}(k, r)$ as the set of cutpaths in $\mathcal{A}$ that leave $k$ cells trough a middles and visit $r$ bounded cells of degree one. From the above considerations we immediately have

$$
|\Gamma(k, r)| \leq\left(\begin{array}{l}
n \\
k
\end{array}\right) 2^{n-k-r} .
$$

With the next lemma we show how to make use of this.

\section{Lemma 2}

$$
|\Gamma(k, r)| \leq \min \left\{\left(\begin{array}{l}
n \\
k
\end{array}\right),\left(\begin{array}{l}
n \\
r
\end{array}\right)\right\} 2^{n-k-r} .
$$



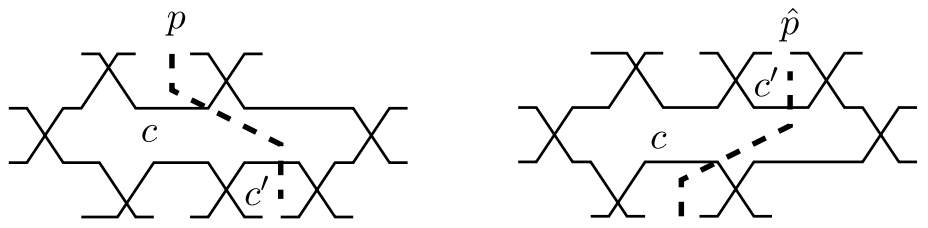

Fig. 7 Illustrating the symmetry between middles and uniques used in Lemma 2

Proof Paths in $\Gamma_{\mathcal{A}}(k, r)$ can also be encoded as cutpaths in the arrangement $\hat{\mathcal{A}}$ obtained from $\mathcal{A}$ via a $180^{\circ}$ rotation of the plane. A cutpath $p$ of $\mathcal{A}$ takes a middle to change from $c$ to $c^{\prime}$ exactly if the rotated cutpath $\hat{p}$ of $\hat{\mathcal{A}}$ is reaching cell $c$ as the unique successor of $c^{\prime}$, see Fig. 7. In other words cells that are left through a middle by $p$ and bounded cells that are left through a unique by $\hat{p}$ are in bijection as well as middles of $\hat{p}$ and bounded uniques of $p$. This yields $\Gamma_{\mathcal{A}}(k, r)=\Gamma_{\hat{\mathcal{A}}}(r, k)$ and the lemma follows from formula (3).

Using the lemma we get

$$
\begin{aligned}
\gamma_{n} & \leq \sum_{k, r}\left|\Gamma_{\mathcal{A}}(k, r)\right| \leq \sum_{k, r} \min \left\{\left(\begin{array}{l}
n \\
k
\end{array}\right),\left(\begin{array}{l}
n \\
r
\end{array}\right)\right\} 2^{n-k-r} \\
& \leq 2^{n} \sum_{k=0}^{n}\left(\begin{array}{l}
n \\
k
\end{array}\right) 2^{-k} \sum_{r \geq k} 2^{-r}+2^{n} \sum_{r=0}^{n}\left(\begin{array}{l}
n \\
r
\end{array}\right) 2^{-r} \sum_{k \geq r} 2^{-k} \\
& =2 \cdot 2^{n} \sum_{k=0}^{n}\left(\begin{array}{l}
n \\
k
\end{array}\right) 2^{-k} \sum_{r \geq k} 2^{-r}=2^{n+1} \sum_{k=0}^{n}\left(\begin{array}{l}
n \\
k
\end{array}\right) 2^{-2 k} \sum_{j \geq 0} 2^{-j} \\
& =2^{n+2}\left(1+\frac{1}{4}\right)^{n}=4\left(\frac{5}{2}\right)^{n} .
\end{aligned}
$$

Combining this with (1) we get:

Theorem 1 The number $B_{n}$ of arrangements of $n$ pseudolines is at most $4^{n-1}\left(\frac{5}{2}\right)\left(\begin{array}{l}n \\ 2\end{array}\right)$, hence for $n$ large enough $b_{n} \leq 0.6609 n^{2}$.

\section{The Upper Bound, Refined}

In this section we show that a careful analysis of the distribution of middles along cutpaths yields an improved bound on the size of $\Gamma(k, r)$.

With an arrangement $\mathcal{A}$ associate the directed dual graph $G_{\mathcal{A}}^{*}$. An example of the underlying undirected dual is shown in Fig. 2. The vertices of $G_{\mathcal{A}}^{*}$ are the cells of $\mathcal{A}$ and the orientation is from north to south. This means that if $\{x, y\}$ is an edge dual to pseudoline $p$ such that $x$ and the north-cell $z_{n}$ are in the same halfplane of $p$ and consequently $y$ and the south-cell $z_{s}$ are in the other halfplane of $p$, then the edge is 

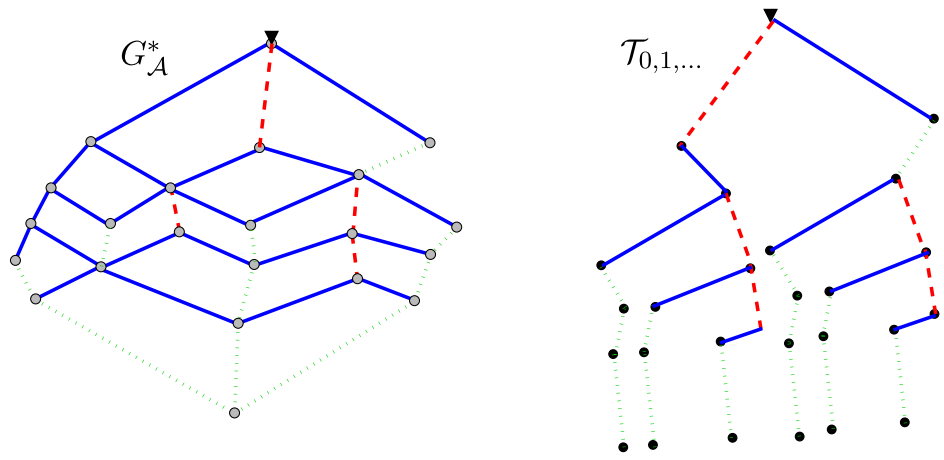

Fig. 8 The graph $G_{\mathcal{A}}^{*}$ and the tree $\mathcal{T}_{\beta}$ with $\beta=0,1, \ldots$, i.e., a right followed by a left

oriented as $(x, y)$. Cutpaths of $\mathcal{A}$ are in bijection to directed $z_{n}$ to $z_{s}$ paths in $G_{\mathcal{A}}^{*}$; henceforth we will use the same name cutpath for these paths in $G_{\mathcal{A}}^{*}$. Edges of $G_{\mathcal{A}}^{*}$ are classified as left, right, middle or unique and they are colored with the label $i$ of their dual pseudoline.

Fix a bitstring $\beta$ and consider the set $\Lambda_{\beta}$ of all cutpaths that can be constructed by using lookup in $\beta$ for the left-right decisions. The paths in $\Lambda_{\beta}(\mathcal{A})$ naturally define a (directed) rooted tree $\mathcal{T}_{\beta}$ : A node $c$ of the tree corresponds to all cutpaths from $\Lambda_{\beta}$ that share a given initial subpath. All edges in $\mathcal{T}_{\beta}$ are oriented away from the root. Corresponding to the tree node $c$ there is a vertex $v_{c}$ in $G_{\mathcal{A}}^{*}$. If $v_{c}$ only has one successor, then tree node $c$ has a unique successor. If $v_{c}$ has more successors, then there is a single sided successor of $c$ representing the cell that is reached with a lookup in $\beta$ and there is a middle successor of $c$ for every middle edge leaving $v_{c}$. Leaves of $\mathcal{T}_{\beta}$ are in bijection to the cutpaths in $\Lambda_{\beta}$. Figure 8 shows an example.

Consider the subtree $\mathcal{T}_{\beta}(r, k)$ of $\mathcal{T}_{\beta}$ consisting of cutpaths in $\Gamma_{\beta}(k, r)=\Lambda_{\beta} \cap$ $\Gamma(k, r)$, i.e., cutpaths using $k$ middle and $r$ unique edges. Let $\mathcal{T}_{\beta}^{*}(r, k)$ be obtained from $\mathcal{T}_{\beta}(r, k)$ by contracting all unique edges. The contractions does not change the number of leaves. Color the sided edge of tree nodes blue and all middle edges red. Note that $\mathcal{T}_{\beta}^{*}(r, k)$ has the following properties:

(a) Every non-leaf node has exactly one blue out-edge.

(b) Every path from the root to a leaf has length $h=n-r$.

(c) Every path from the root to a leaf uses exactly $k$ red edges.

(d) The nodes along any path from the root to a leaf have altogether at most $n$ red out-edges (Lemma 1).

Definition 1 For $n \geq h \geq k$, let $T(n, h, k)$ be the maximum number of leaves of a rooted tree with red and blue edges and properties (a)-(d).

To determine this quantity we first study a different maximization problem. A $k$ transversal of a partition $\Pi=\left(B_{1}, \ldots, B_{h}\right)$ of $[n]$ is a $k$-element subset $A$ of $[n]$ such that $\left|A \cap B_{i}\right| \leq 1$ for each $i \in\{1, \ldots, h\}$. 
Definition 2 For $n \geq h \geq k$, let $P(n, h, k)$ be the maximum number of $k$-transversals a partition $\Pi=\left\{B_{1}, \ldots, B_{h}\right\}$ of $[n]$ with $h$ blocks can have.

Lemma 3 If $n=d \cdot h+r$ with $0 \leq r<h$, then

$$
P(n, h, k)=\sum_{\ell=0}^{r}\left(\begin{array}{l}
r \\
\ell
\end{array}\right)(d+1)^{\ell}\left(\begin{array}{l}
h-r \\
k-\ell
\end{array}\right) d^{k-\ell} .
$$

Proof Let $\Pi^{*}$ be a partition of [n] into $r$ blocks of size $d+1$ and $h-r$ blocks of size $d$. It is an easy exercise to show that the number of $k$-transversals of $\Pi^{*}$ is equal to the right hand side of the equation in the lemma.

Now consider a partition $\Pi$ of $[n]$ into $h$ blocks and suppose it is not equivalent to $\Pi^{*}$. Then there are blocks $B$ and $B^{\prime}$ in $\Pi$ whose size differs by at least two. Let $|B|=s$ and $\left|B^{\prime}\right|=s+t$ with $t \geq 2$. Now let $\hat{\Pi}$ be obtained by moving one element from $B^{\prime}$ to $B$ so that $\hat{\Pi}$ has blocks $\hat{B}$ and $\hat{B}^{\prime}$ with $|\hat{B}|=s+1$ and $\left|\hat{B}^{\prime}\right|=s+t-1$. Note that the $k$-transversals of $\Pi$ and $\hat{\Pi}$ whose intersection with $B \cup B^{\prime}$ is at most one are the same. Now let $M$ be the number of $(k-2)$-transversals of $\Pi \backslash\left\{B, B^{\prime}\right\}$. The number of $k$-transversals whose intersection with $B \cup B^{\prime}$ is two is $s(s+t) M$ for $\Pi$ and $(s+1)(s+t-1) M$ for $\hat{\Pi}$. Since $(s+1)(s+t-1)>s(s+t)$ it follows that $\hat{\Pi}$ has more $k$-transversals than $\Pi$.

Lemma 4 For all $k \leq h \leq n$,

$$
P(n, h, k) \leq\left(\begin{array}{l}
h \\
k
\end{array}\right)\left(\frac{n}{h}\right)^{k} .
$$

Proof Let $\Pi$ be a partition of $[n]$ into $h$ blocks whose number of $k$-transversals is exactly $P(n, h, k)$. From $\Pi$ we obtain a partition $\Pi^{+}$of $[h n]$ into $h$ blocks by substituting single elements by sets of $h$ elements, e.g., $i \rightarrow(i-1) h+1, \ldots, i h$.

The number of $k$-transversals of $\Pi^{+}$is $h^{k} P(n, h, k)$. With Lemma 3 we get the result: $h^{k} P(n, h, k) \leq P(h n, h, k)=\left(\begin{array}{l}h \\ k\end{array}\right) n^{k}$.

Lemma $5 T(n, h, k) \leq P(n, h, k)$.

Proof The proof is by induction on $h$. Since $k \leq h$ we get a start by verifying $P(n, 1,0)=T(n, 1,0)=1$.

For the inductive step consider a tree realizing $T(n, h+1, k)$. The root of the tree has some degree $s+1$. It follows from the defining properties (a)-(d) that the subtree reached from the root through the blue edge contains at most $T(n-s, h, k)$ leaves and each of the $s$ subtrees reached from the root through a red edge contains at most $T(n-s, h, k-1)$ leaves. Therefore,

$$
T(n, h+1, k) \leq \max _{0 \leq s \leq n-k+1}(T(n-s, h, k)+s T(n-s, h, k-1)) .
$$

Now let $s$ be the value where the maximum is attained. By induction

$$
T(n-s, h, k)+s T(n-s, h, k-1) \leq P(n-s, h, k)+s P(n-s, h, k-1) .
$$


Consider partitions $\Pi_{1}$ and $\Pi_{2}$ maximizing $P(n-s, h, k)$ and $P(n-s, h, k-1)$. A byproduct of the proof of Lemma 3 is that for $k \geq 2$ the structure of the maximizing partitions is independent of $k$. For $k=0,1$ all partitions with $h$ blocks are maximizing. Hence, we can assume that $\Pi_{1}=\Pi_{2}$. Let $\Pi$ be the partition obtained by adding a new block $B$ with $|B|=s$ to $\Pi_{1}$ and note that the right hand side of (6) is exactly the number of $k$-transversals of $\Pi$. Since $\Pi$ is a partition of an $n$-element set into $h+1$ blocks we conclude

$$
P(n-s, h, k)+s P(n-s, h, k-1) \leq P(n, h+1, k) .
$$

This completes the proof.

We mention without proof that the inequality in Lemma 5 actually holds with equality.

To bound the total number $\gamma_{n}$ of cutpaths in an arrangement $\mathcal{A}$, we use the ideas developed in this section in a series of inequalities:

$$
\begin{aligned}
\gamma_{n} & \leq \sum_{k, r}\left|\Gamma_{\mathcal{A}}(k, r)\right|=\sum_{k, r} \sum_{\beta}\left|\Lambda_{\beta} \cap \Gamma_{\mathcal{A}}(k, r)\right| \\
& =\sum_{k, r} \sum_{\beta} \# \text { leaves of } \mathcal{T}_{\beta}(k, r) \leq \sum_{k, r} \sum_{\beta} T(n, n-r, k) \\
& =\sum_{k, r} T(n, n-r, k) 2^{n-r-k} \leq \sum_{k, r} P(n, n-r, k) 2^{n-r-k} .
\end{aligned}
$$

Recall from Lemma 2 that the freedom of encoding cutpaths forward or backward yields a symmetry in the parameters $k$ and $r$. Using this and the observation that $h>h^{\prime}$ implies $P(n, h, k)>P\left(n, h^{\prime}, k\right)$ we get:

$$
\begin{aligned}
\gamma_{n} & \leq \sum_{k, r} \min \{P(n, n-r, k), P(n, n-k, r)\} 2^{n-r-k} \\
& \leq \sum_{k \leq r} 2 P(n, n-r, k) 2^{n-r-k} \leq \sum_{k} 2 P(n, n-k, k) 2^{n-2 k} \sum_{j \geq 0} 2^{-j} \\
& =4 \sum_{k}\left(\begin{array}{c}
n-k \\
k
\end{array}\right)\left(\frac{n}{n-k}\right)^{k} 2^{n-2 k} .
\end{aligned}
$$

The last equality follows from Lemma 4.

Now we are interested in the summand of (9) whose contribution is asymptotically dominant. Using Stirling's approximation and parameterizing $k=a n$, the summands can be estimated as

$$
\left(\begin{array}{c}
(1-a) n \\
a n
\end{array}\right)\left(\frac{n}{(1-a) n}\right)^{a n} 2^{(1-2 a) n} \approx\left[2\left(\frac{1-a}{1-2 a}\right)^{(1-2 a)}\left(\frac{1}{4 a}\right)^{a}\right]^{n} .
$$

At this point we started Maple and found that presumably the maximum of (10) is attained at $a \approx 0.186691$ and has the value of at least 2.486976 , so that $\gamma_{n} \leq$ $4 n 2.486976^{n}$. This yields our main theorem: 

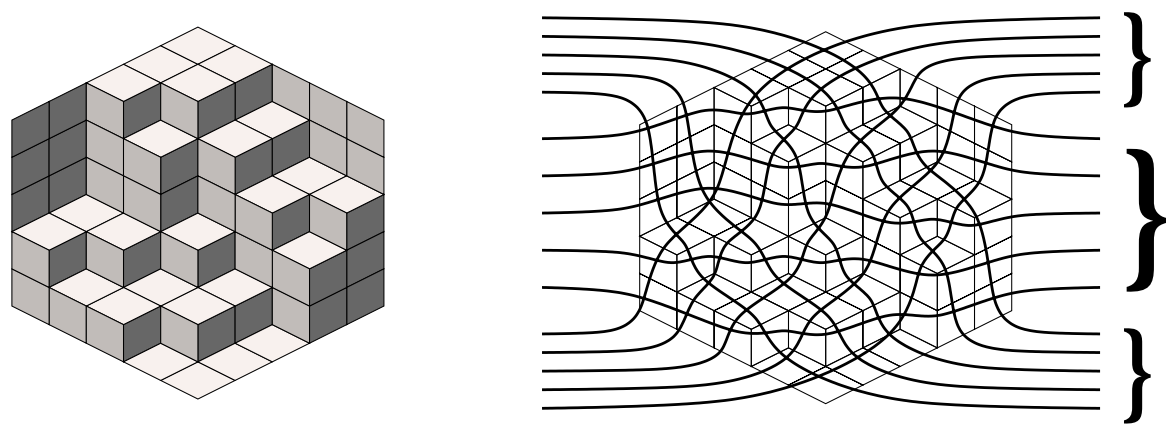

Fig. 9 The hexagon $H(5,5,5)$ with one of its rhombic tilings and a consistent partial arrangement corresponding to the tiling

Theorem 2 Let $B_{n}$ be the number of arrangements of $n$ pseudolines and let $b_{n}=$ $\log _{2} B_{n}$. For $n$ large enough, $b_{n} \leq 0.6571 n^{2}$.

\section{A Lower Bound}

Given three numbers $i, j$ and $k$ we consider the set of $i+j+k$ pseudolines $1,2, \ldots, i+j+k$ partitioned into the following three parts: $\{1, \ldots, i\},\{i+1, \ldots, i+$ $j\}$, and $\{i+j+1, \ldots, i+j+k\}$. A partial arrangement on this set is called consistent if any two pseudolines from different parts cross while any two pseudolines from the same part do not cross. The zonotopal duals of consistent partial arrangements are rhombic tilings of the centrally symmetric hexagon $H(i, j, k)$ with side lengths $i, j$ and $k$; Fig. 9 shows an example.

The enumeration of rhombic tilings of $H(i, j, k)$ is a classical combinatorial problem solved by MacMahon [10]. There are

$$
P P(i, j, k)=\prod_{a=0}^{i-1} \prod_{b=0}^{j-1} \prod_{c=0}^{k-1} \frac{a+b+c+2}{a+b+c+1}
$$

such tilings.

Consider a consistent partial arrangement with three parts of size $n$. Such a partial arrangement can be completed to a 'full' arrangement of $3 n$ pseudolines by adding any arrangement of $n$ lines for each of the three parts. E.g. in Fig. 9 the addition can be done by gluing three arrangements of five pseudolines each to the picture where the braces are. This construction shows that

$$
B_{3 n} \geq P P(n, n, n) B_{n}{ }^{3} \text {. }
$$

To find the growth rate of $P P(n, n, n)$ we first note that $P P(n, n, n)=T(n) / T(0)$ where $T(k)=\prod_{a=0}^{n-1} \prod_{b=0}^{n-1}(a+b+k+1)$. Let $t(k)=\ln T(k)$ and approximate $t(k)$ 
by an integral:

$$
t(k)=\ln T(k)=\sum_{a=0}^{n-1} \sum_{b=0}^{n-1} \ln (a+b+k+1) \approx \int_{x=0}^{n} \int_{y=0}^{n} \ln (x+y+k+1) d y d x .
$$

From this approximation it can be concluded that

$$
\ln P P(n, n, n)=t(n)-t(0) \approx\left(\frac{9}{2} \ln (3)-6 \ln (2)\right) n^{2} .
$$

Combining this with Formula (12) we get

Proposition 1 The number $B_{n}$ of arrangements of $n$ pseudolines is at least $2^{0.1887 n^{2}}$.

Computations were mainly done with Maple.

Acknowledgements S. Felsner is partially supported by DFG grant FE-340/7-1.

Work by P.V. was supported by the projects 1M0545 and MSM0021620838 of the Ministry of Education of the Czech Republic.

\section{References}

1. Bílka, O.: On the number of ways to add a pseudoline. Internal Report (2010)

2. Björner, A., Las Vergnas, M., White, N., Sturmfels, B., Ziegler, G.M.: Oriented Matroids. Cambridge University Press, Cambridge (1993)

3. Felsner, S.: On the number of arrangements of pseudolines. Discrete Comput. Geom. 18, 257-267 (1997)

4. Felsner, S.: Geometric Graphs and Arrangements. Advanced Lectures in Mathematics. Vieweg, Wiesbaden (2004)

5. Fishburn, P.: Acyclic sets of linear orders. Soc. Choice Welf. 14, 113-124 (1997)

6. Galambos, Á., Reiner, V.: Acyclic sets of linear orders via the Bruhat orders. Soc. Choice Welf. 30, 245-264 (2008)

7. Goodman, J.E.: Proof of a conjecture of Burr, Grünbaum and Sloane. Discrete Math. 32, 27-35 (1980)

8. Goodman, J.E.: Pseudoline arrangements. In: Goodman, J.E., O’Rourke, J. (eds.) Handbook of Discrete and Computational Geometry, pp. 83-110. CRC Press, Boca Raton (1997)

9. Knuth, D.E.: Axioms and Hulls. Lect. Notes Comput. Sci., vol. 606. Springer, Berlin (1992)

10. MacMahon, P.A.: Combinatory Analysis, vol. II. Chelsea, New York (1960). Reprint of the 1916 edition

11. Matoušek, J.: Lectures on Discrete Geometry. Graduate Texts in Mathematics, vol. 212. Springer, Berlin (2002)

12. Ziegler, G.M.: Higher Bruhat orders and cyclic hyperplane arrangements. Topology 32, 259-279 (1993) 